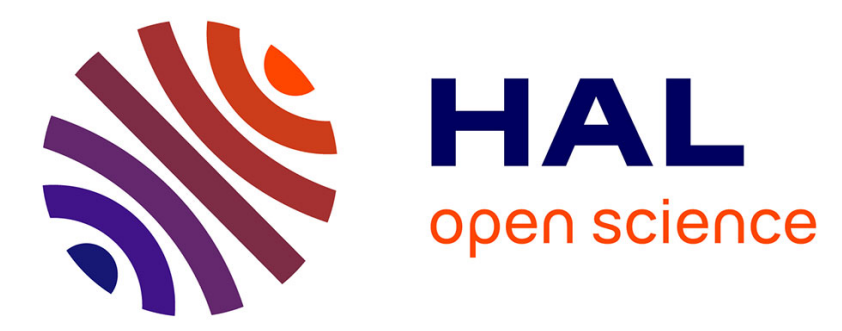

\title{
Théorie des styles explicatifs et performance sportive: fondements théoriques, données empiriques et perspectives
}

\author{
Charles Martin-Krumm, Philippe Sarrazin
}

\section{To cite this version:}

Charles Martin-Krumm, Philippe Sarrazin. Théorie des styles explicatifs et performance sportive: fondements théoriques, données empiriques et perspectives. Science et Motricité: revue scientifique de l'ACAPS / ACAPS, 2004, 52, pp.9-43. 10.3917/sm.052.0009 . hal-00388566

\section{HAL Id: hal-00388566 https://hal.science/hal-00388566}

Submitted on 27 May 2009

HAL is a multi-disciplinary open access archive for the deposit and dissemination of scientific research documents, whether they are published or not. The documents may come from teaching and research institutions in France or abroad, or from public or private research centers.
L'archive ouverte pluridisciplinaire HAL, est destinée au dépôt et à la diffusion de documents scientifiques de niveau recherche, publiés ou non, émanant des établissements d'enseignement et de recherche français ou étrangers, des laboratoires publics ou privés. 


\title{
Théorie des styles explicatifs et performance sportive : fondements théoriques, données empiriques et perspectives
}

\author{
Charles Martin-Krumm ${ }^{(1)}$, Philippe Sarrazin ${ }^{(2)}$
}

\section{RÉSUMÉ}

Cette revue de littérature porte sur la théorie des "styles attributionnels » ou "styles explicatifs » (e.g., Abramson, Seligman E Teasdale, 1978), selon laquelle, chaque individu aurait une manière relativement stable d'expliquer les événements positifs ou négatifs susceptibles de lui arriver. Malgré son intérêt, celle-ci n'a fait l'objet que de très peu d'études dans le domaine des activités physiques et sportives. L'objectif de cet article est de faire un état des lieux de cette théorie, et de montrer en quoi elle peut intéresser la recherche dans le domaine du sport ou de l'éducation physique. Seront abordés en particulier, les racines historiques de la théorie, le problème de la mesure du style explicatif, les conséquences affectives, cognitives et comportementales des styles dans le domaine de la performance sportive, et la question de leurs antécédents. Enfin, les perspectives actuelles de ce champ théorique seront développées. Nous montrerons qu'après avoir été considéré comme un corrélat de différents symptômes, le style est maintenant appréhendé comme une variable distale et/ou une variable modulatrice. Les travaux portent maintenant davantage sur les processus, ainsi les résultats permettent de mieux comprendre les relations entre le style explicatif et différents types de déficits.

Mots-clés : style explicatif, optimisme, pessimisme, performance, motivation, médiation, modulation.

(1) Université Paris XI, Bâtiment 335, 91405 Orsay Cedex, et IFEPS, Angers, 49 rue des Perrins, 49130 Les Ponts-de-Cé.

(2) Laboratoire Sport et Environnement Social, UFRAPS, Université J. Fourier Grenoble 1, BP 53-38041 Grenoble Cedex 9. 


\title{
The attributional style theory and sport performance : Theoretical backgrounds, empirical data and future directions
}

\begin{abstract}
This literature review aims at presenting the "attributional style (Abramson, Seligman $\mathcal{E}$ Teasdale, 1978) or "explanatory style » theory (Buchanan E Seligman, 1995) according to which each individual would have a relative stable manner to explain the positive or negative events which may happen to him/her. Despite its interest, there are only very few studies about this theory in the field of sports. The purpose of this article is to draw up the most recent works based on this theory and to show how it may interest research in the field of sports or physical education. More generally, (1) the historical roots of this theory, (2) the problem of the measure of explanatory style, (3) the affective, cognitive and behavioral consequences of the styles in the field of sports performance, and (4) their antecedents will be tackled. Finally, current perspectives of this theoretical area will be developed. We will show that the explanatory style, after having been considered as a correlate for various symptoms, is now rather considered as a distal variable and/or a moderating variable. Thus, studies now rather deal with processes which allow a better comprehension about the links between explanatory style and different kinds of deficits.
\end{abstract}

Key-words : explanatory style, optimism, pessimism, performance, motivation, mediation, moderation.

Le sport et l'école sont des domaines d'accomplissement dans lesquels les trajectoires des uns et des autres dépendent des succès, mais aussi des échecs vécus. Pourtant, force est de constater des différences singulières dans la manière dont les individus réagissent. Après un échec cuisant ou une blessure sévère, certains vont s'effondrer, se sentant incapables de revenir sur le devant de la scène. D'autres, au contraire, vont « rebondir» et considérer l'échec comme un obstacle temporaire, ouvrant la porte à de nouveaux défis.

L'Homme naïf qualifierait les premiers de pessimistes, et les seconds — capables de " positiver » les situations les plus critiques - d'optimistes. Mais qu'est-ce que l'optimisme et le pessimisme ? S'agit-il de caractéristiques personnelles générales et relativement endurantes, ou d'attributs plus labiles et spécifiques aux situations ? Comment devienton optimiste ou pessimiste? Quelles en sont les conséquences affectives, 
cognitives et comportementales? Et par l'intermédiaire de quels mécanismes ? Apporter des réponses à de telles questions présente un intérêt considérable pour l'entraîneur comme pour l'enseignant. Mieux comprendre ce phénomène permettrait à la fois de repérer les élèves et les athlètes à risque élevé de schémas dysfonctionnels en cas d'échec, et de mettre en œuvre des stratégies susceptibles de réduire le développement de personnalités pessimistes.

Une théorie particulièrement à même d'éclairer cette problématique est la théorie des styles attributionnels ou des styles explicatifs (e.g., Abramson, Seligman \& Teasdale, 1978). Elle tire ses racines de la théorie de la résignation apprise (learned helplessness). Selon cette théorie, lorsqu'un individu échoue devant une situation dans laquelle il ne perçoit aucun moyen d'atteindre le but, celui-ci fait l'apprentissage de l'inutilité de ses efforts et peut devenir résigné. Selon Gernigon, Fleurance, Wolff et Reine (1998), la résignation apprise correspond alors à une perception d'indépendance entre comportements et résultats, à l'origine d'une expectation d'incontrôlabilité qui peut s'étendre au-delà même de la situation qui en a généré l'apprentissage. Par la suite, des éléments empruntés à la théorie de l'attribution causale (e.g., Weiner, 1974) ont donné lieu à une reformulation de la théorie de la résignation apprise (Abramson et al., 1978), mettant l'accent sur la manière dont l'individu explique les événements auxquels il est confronté. Le concept de «style attributionnel » ou « style explicatif » était né. Les recherches autour de ce concept se sont progressivement émancipées des problèmes stricts de résignation apprise et contribuent aujourd'hui aux problématiques relatives à l'orientation optimiste ou pessimiste d'une personne (e.g., Chang, 2000).

Malgré son intérêt, la théorie des styles explicatifs n'a fait l'objet que de très peu d'études dans le domaine du sport ou de l'école. L'un des objectifs de cette revue de littérature est précisément de faire une recension des travaux utilisant cet éclairage théorique dans le domaine du sport et des activités physiques. Plus généralement, seront traitées les questions relatives (1) à la mesure du style, (2) à ses conséquences affectives, cognitives ou comportementales, et (3) à ses antécédents. Enfin, les orientations actuelles de ce cadre théorique seront évoquées. Nous montrerons en particulier, qu'après avoir été considéré comme une variable proximale, le style est maintenant plutôt considéré comme une variable distale - un " facteur de risque » (e.g., Peterson \& Seligman, 1984). Aussi les travaux actuels (1) cherchent à identifier le rôle de « médiateurs » potentiels entre cette caractéristique personnelle et cer- 
taines variables comportementales, ou (2) considèrent le style comme une variable " modulatrice », supposée accentuer ou inhiber l'influence d'autres variables.

Dans une première partie, une présentation de la théorie des styles sera effectuée. Après avoir rappelé les racines historiques de celle-ci, sera abordé dans une deuxième partie le problème de sa mesure et dans une troisième partie, celui de la définition de l'optimisme et du pessimisme. La quatrième partie de cette contribution, présentera les résultats des études sur les conséquences des styles, en particulier en contexte sportif ; sera également abordé le «statut » de cette variable - à savoir - simple corrélat, variable distale ou variable modulatrice. La cinquième partie s'attachera plus particulièrement aux travaux sur les antécédents du style et sur la manière dont cette caractéristique personnelle se cristallise chez l'enfant. Enfin la conclusion permettra de présenter les perspectives de recherches sur la théorie des styles explicatifs.

\section{De la Résignation Apprise au style explicatif}

La résignation apprise a été étudiée pour la première fois sur des chiens (e.g., Overmier \& Seligman, 1967). Le protocole consistait, lors d'une phase de pré-traitement, à soumettre des chiens à des chocs électriques auxquels ils ne pouvaient pas échapper. Ensuite, lors d'une phase de test, les chiens étaient placés dans une cage et subissaient à nouveau des chocs électriques, mais cette fois-ci ils avaient la possibilité d'y échapper à partir d'une réponse simple. Les chiens ayant subi cette phase de prétraitement n'apprenaient pas cette réponse. Au lieu de cela, ils adoptaient des attitudes prostrées et résignées, et subissaient passivement la décharge électrique. Cette attitude contrastait fortement avec celle de chiens n'ayant pas subi la phase de pré-traitement, qui réagissaient aux chocs et apprenaient rapidement comment y mettre fin.

Selon les chercheurs, les chiens avaient «appris » à être résignés ; après l'exposition préalable à un choc incontrôlable, ils apprenaient qu'il n'y avait plus rien à faire : le choc électrique surviendrait, et cela indépendamment de leurs comportements (Maier \& Seligman, 1976). Autrement dit, il est présumé que l'apprentissage de l'indépendance du lien entre les comportements et les résultats est représenté sous la forme d'une attente de résignation susceptible de se généraliser à de nouvelles situations et de produire trois types de déficits : motivationnel, cognitif et émotionnel. Le premier se traduit par de la passivité, le second par la 
difficulté d'apprendre que certaines réponses de l'individu conduisent à des résultats, et le dernier par des états émotionnels négatifs.

Après avoir été observé chez d'autres espèces animales, ce phénomène a commencé à être étudié chez l'Homme. Le protocole expérimental était le même que celui utilisé pour l'animal : les sujets étaient confrontés à des événements incontrôlables dont les conséquences étaient observées. L'exposition à des bruits désagréables ou à des problèmes insolubles (e.g., Hiroto \& Seligman, 1975 ; Seligman, 1975) a remplacé les décharges électriques, mais les effets du phénomène subsistaient: après avoir fait l'expérience d'incontrôlabilité (i.e., de l'inutilité de leurs efforts), les personnes manifestent une variété de déficits motivationnels, cognitifs ou émotionnels (pour une revue de ces études voir, Mikulincer, 1994 ; Peterson, Maier \& Seligman, 1993).

Dans le domaine du sport, quelques rares études se sont intéressées aux conséquences de l'induction d'incontrôlabilité sur la performance motrice. Par exemple, Gernigon, Fleurance et Reine (2000) ont effectué deux expériences utilisant une tâche de tir au pistolet. Dans ce qu'ils ont d'essentiel, les résultats montrent que les participants confrontés dans un premier temps à une situation dans laquelle les feed-back de réussite ou d'échec sont indépendants de l'atteinte ou non de la cible (tâche incontrôlable), sont généralement moins persistants (en terme de nombre d'essais tentés) et moins performants dans une tâche subséquente identique, par rapport à ceux qui ont été confrontés préalablement à une situation contrôlable (dans laquelle les feed-back de réussite ou d'échec dépendent de l'atteinte ou non de la cible).

Plusieurs critiques ont commencé à s'élever parallèlement à l'avancée des recherches sur la résignation apprise. Certains chercheurs ont dénoncé le manque de validité interne de certaines expériences, en apportant d'autres explications aux baisses de performance constatées dans celles-ci (pour une revue voir, Ric, 1996, 2001). Par exemple, la réaction aux feed-back d'échec qui conduisaient les sujets à utiliser une stratégie de protection de soi (e.g., Frankel \& Snyder, 1978), la focalisation de l'attention du sujet sur des éléments qui ne concernent pas la tâche à proprement dite (e.g., Mikulincer \& Nizan, 1988), 1'hostilité éprouvée envers l'expérimentateur étant donné le caractère aversif de certaines manipulations expérimentales (e.g., Silver, Wortman \& Klos, 1982), semblent constituer autant d'hypothèses explicatives alternatives à celle de l'apprentissage d'incontrôlabilité ou de l'inutilité de l'effort. D'autre part, la validité même de la théorie a été remise en cause. Le 
modèle ne permet pas d'expliquer l'ensemble des réactions que les individus démontrent face aux événements incontrôlables (voir les synthèses faites par, Miller \& Norman, 1979 ; Roth, 1980 ; Wortman \& Brehm, 1975) : certains manifestent les déficits présumés à travers le temps et les situations, tandis que d'autres ne manifestent pas ces déficits. Par exemple, l'absence de contrôle sur le tirage des boules au Loto n'entraîne pas pour autant des déficits motivationnels, cognitifs ou émotionnels chez les joueurs. Ainsi, le modèle de la résignation apprise dans sa formulation originale semble constituer une simplification excessive quand il est appliqué à l'être humain (Peterson \& Park, 1998).

C'est la raison pour laquelle, Abramson, Seligman et Teasdale (1978) ont reformulé le modèle de la résignation apprise en empruntant des éléments à la théorie de l'attribution (Kelley, 1973 ; Weiner, 1974), afin de le rendre plus compatible au fonctionnement humain. Pour résoudre les résultats contradictoires observés dans la littérature, Abramson et al. ont fait évoluer la théorie en prenant en compte la manière dont la personne explique ou interprète son manque de contrôle sur une situation. La nature de sa réponse - l'attribution causale qu'il formule - donne alors les caractéristiques de la résignation qui suit. Si l'attribution causale est stable ( « cela va durer longtemps car quoi que je fasse, rien ne changera »), elle induira une résignation chronique ; si elle est instable, alors la résignation sera transitoire. D'autre part, si l'attribution causale est globale («c'est pour tout pareil»), alors les symptômes de résignation seront susceptibles de se généraliser à des domaines variés (i.e., résignation apprise globale). Par contre, si les causes sont spécifiques, alors les symptômes seront circonscrits à un domaine particulier (i.e., résignation apprise spécifique). Enfin, la perception d'un manque de contrôle attribuée à une cause interne (" c'est entièrement de ma faute »), entraînera une diminution de l'estime de soi (i.e., résignation apprise personnelle), ce qui ne sera pas le cas, si la cause invoquée est externe (" c'est très difficile »)(i.e., résignation apprise universelle).

Ces hypothèses, résumées dans la figure 1 , sont à la base de la reformulation "attributionnelle » de la théorie de la résignation apprise (Abramson et al., 1978). Cette nouvelle formulation de la théorie ne change pas les postulats du modèle original : les événements incontrôlables sont toujours présumés conduire à des déficits quand ils engendrent une attente d'indépendance entre les actions de l'individu et les résultats obtenus. Cependant, la nature de ces déficits dépend à présent de l'attribution causale émise par l'individu. 


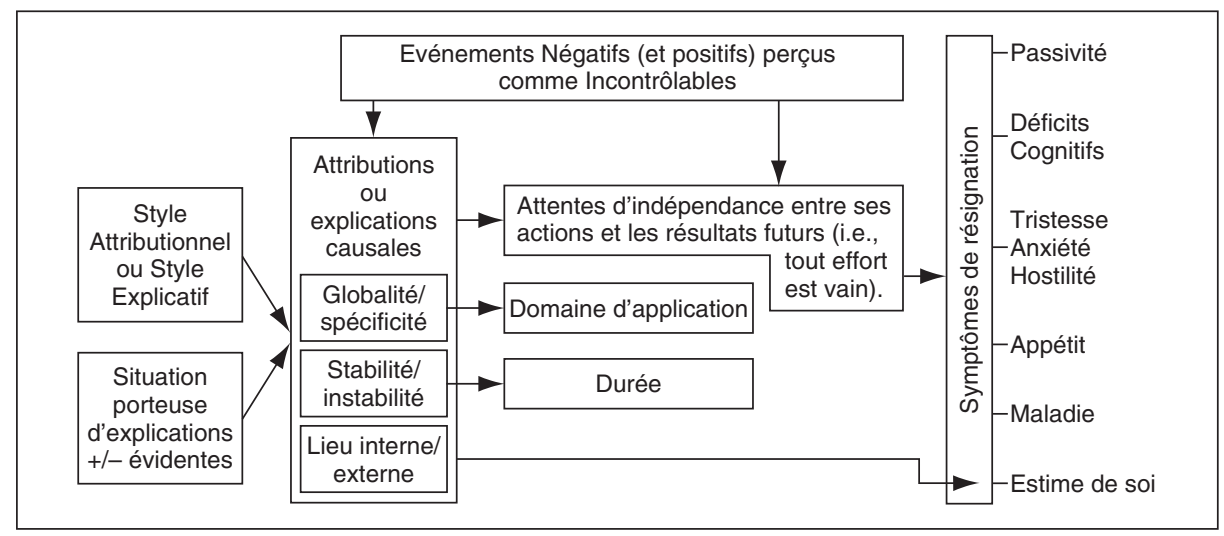

FIGURE I

Le processus de la résignation apprise (inspiré de Peterson \& Seligman, 1984, p. 350).

Dans certains cas, la situation en elle-même est porteuse d'explications des réussites ou des échecs rencontrés. La littérature sur les attributions causales souligne la variété des influences situationnelles du processus (e.g., Shaver, 1975). Par exemple, une défaite au volley-ball contre une équipe classée deux ou trois divisions au dessus aurait de fortes chances d'être attribuée au niveau de cette équipe, et pas à l'incompétence des partenaires. Dans d'autres cas, l'individu utilise des schémas explicatifs stables et récurrents, qu'Abramson et al. (1978) ont appelé style attributionnel ou style explicatif (attributional style). Quand un événement incontrôlable survient, et quand les explications situationnelles ne sont pas évidentes, alors le style explicatif devrait influencer la manière dont l'individu va se comporter. La résignation sera chronique ou transitoire, globale ou spécifique, préjudiciable à l'estime de soi ou non, en fonction précisément du style explicatif de l'individu (Peterson \& Park, 1998).

En résumé, le style explicatif est « la tendance d'une personne à donner le même type d'explications aux différents événements auxquels elle est confrontée » (Peterson, Buchanan \& Seligman, 1995, p. 1). C'est une manière relativement stable d'expliquer — en terme d'internalité ${ }^{(3)}$, de

(3) Dans les derniers articles empiriques et théoriques (e.g., Abramson, Metalsky \& Alloy, 1989 ; Dykema, Bergbower, Doctora \& Peterson, 1996 ; Peterson, 1991 ; Peterson, Bishop, Fletcher, Kaplan, Yesko, Moon, Smith, Michaels \& Michaels, 2001 ; Peterson \& Vaidya, 2001), la dimension internalité a progressivement été délaissée car sa mesure semble moins fiable, et ses corrélats (avec les expectations par exemples) moins consistants, que ceux obtenus avec la stabilité ou la globalité. 
stabilité, et de globalité - une variété d'événements négatifs ou positifs qui surviennent et dans lesquels l'individu est impliqué. Il s'agit par exemple de l'incapacité à suivre un entraînement, une lourde défaite, une réprimande de l'entraîneur, une dispute avec les partenaires, une victoire importante pour la suite de la saison sportive etc.

\section{Mesurer le style explicatif}

Le style explicatif a engendré des recherches propres à partir du moment où ont commencé à se développer des mesures de cette propension personnelle. Plusieurs outils existent à présent, et vont être présentés rapidement (pour une discussion plus détaillée sur ces mesures, voir Reivich, 1995).

La première mesure de style explicatif qui a été développée, est le Questionnaire de Style Attributionnel (Attributional Style Questionnaire ou ASQ ; Peterson, Semmel, von Baeyer, Abramson, Metalsky \& Seligman, 1982 ; Seligman, Abramson, Semmel \& von Baeyer, 1979). L'ASQ demande aux sujets d'imaginer qu'ils soient confrontés à un ensemble d'événements hypothétiques positifs (e.g., "Imaginez que vous obteniez une promotion ") et négatifs (e.g., "Imaginez qu'un de vos amis vous dise qu'il ne peut pas avoir confiance en vous ») pour lesquels ils doivent à chaque fois mentionner la « cause principale » de celui-ci. Ces scénarii mettent en scène des événements susceptibles de survenir dans le contexte large de l'accomplissement (succès ou échecs professionnels ou scolaires) et de l'affiliation. Ensuite, les sujets doivent indiquer sur une échelle en 7 points pour chaque scénario, le degré avec lequel la cause est interne versus externe (e.g., « Est-ce que la cause du manque de confiance de votre ami est due à quelque chose qui vient de vous ou à quelque chose qui vient d'autres personnes ou des circonstances?), stable versus instable (e.g., " Dans le futur quand vous interagirez avec vos amis, est-ce que cette cause sera toujours présente?»), et globale versus spécifique («Est-ce cette cause est quelque chose qui influence uniquement vos interactions avec vos amis, ou est-elle susceptible d'influencer d'autres domaines de votre vie?»).

Plusieurs variantes de l'ASQ existent. Certains outils ne proposent que des scenarii négatifs (e.g., Expanded-ASQ de Peterson \& Villanova, 1988 ; ASQ for general use de Dykema, Bergbower, Doctora \& Peterson, 1996), d'autres outils ont adopté un mode de réponse à choix forcé pour pallier certaines difficultés rencontrées - en particulier avec les jeunes 
sujets - pour compléter le type de réponse aux questions de l'ASQ (e.g., Children's ASQ ; Kaslow, Tannenbaum \& Seligman, 1978 ; ForcedChoise ASQ ; Reivich \& Seligman 1991).

Si la conceptualisation du style comme un trait de personnalité semble largement reconnue, certains théoriciens (e.g., Cutrona, Russell \& Jones, 1985 ; Peterson \& Park, 1998 ; Peterson, 2000) préconisent néanmoins d'effectuer une évaluation de ce construit qui soit relativement spécifique à un domaine particulier. C'est la raison pour laquelle sont apparus récemment des outils similaires à l'ASQ, mais spécifiquement conçus pour le domaine scolaire (e.g., Academic ASQ ; Peterson \& Barrett, 1987) ou celui de la finance (e.g., Financial Service ASQ ; Proudfoot, Corr, Guest \& Gray, 2001). Ainsi, pour évaluer le style explicatif des athlètes, certains auteurs (e.g., Biddle, Hanrahan \& Sellars, 2001) recommandent l'utilisation d'une mesure spécifique au sport, comme l'Échelle du Style Explicatif en Sport (Sport Attributional Style Scale; SASS) dans sa version longue (Hanrahan, Grove \& Hattie, 1989) ou courte (Harahan \& Grove, 1990), ou sa version modifiée et validée en français: l'Échelle Sportive de Mesure du Style Explicatif (ÉSMSE; Martin-Krumm, Sarrazin, Fontayne \& Famose, 2001), plutôt qu'une mesure trop générale. L'ÉSMSE comprend 10 situations hypothétiques fréquentes dans le domaine du sport : 5 scénarii positifs (e.g., " vous faites une bonne performance à une compétition"; "vos coéquipiers disent de vous que vous êtes un bon athlète ») et 5 scénarii négatifs (le contraire de chaque scénario positif). Comme pour l'ASQ, les participants doivent fournir la cause la plus probable à chaque événement, et spécifier ensuite le degré de stabilité et de globalité de celle-ci sur une échelle en 7 points (voir le Tableau 1 pour un exemple d'item). La dimension locus de causalité a été abandonnée à la fois pour des raisons métrologiques et théoriques (note 1; Dykema et al., 1996 ; Peterson, 2000 ; Peterson \& Vaidya, 2001).

Une analyse factorielle confirmatoire effectuée à partir des réponses de plus de 600 adolescents (Martin-Krumm et al., 2001) a fourni un appui fort à l'existence de deux dimensions corrélées (i.e., stabilité et globalité des causes), à la fois pour les événements positifs et les événements négatifs. Par ailleurs, la fiabilité de l'échelle s'est révélée fort satisfaisante, que ce soit au niveau de sa consistance interne (coefficients alphas de Cronbach de chaque sous échelle $\geq .70$ ) ou de sa stabilité temporelle (corrélations test-restest sur 6 mois $\geq .40$ ). 
TABLEAU I

Exemple d'événement tiré de l'Échelle Sportive de Mesure du Style Explicatif

Imaginez que vous réalisiez une très bonne performance lors d'une compétition sportive.

1. Écrivez ci-dessous, la cause la plus probable, selon vous, de votre bonne performance :

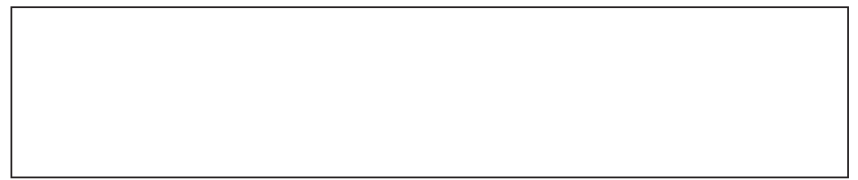

2. Si dans le futur vous réalisez à nouveau de très bonnes performances sportives, pensez-vous que la cause (que vous avez notée dans le rectangle ci-dessus) sera la même? (Encerclez un chiffre)

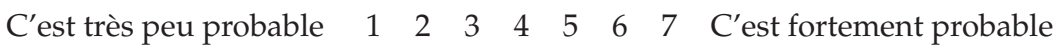

3. Est-ce que la cause, que vous avez mentionnée dans le rectangle ci-dessus, est quelque chose qui permet d'expliquer uniquement vos bonnes performances sportives, ou est-ce quelque chose susceptible d'influencer aussi d'autres aspects de votre vie (par exemple vos performances scolaires, vos relations avec les autres, votre vie sentimentale etc.) ? (Encerclez un chiffre)

Permet d'expliquer seulement cet événement particulier de ma vie

$$
\begin{array}{llllllll}
1 & 2 & 3 & 4 & 5 & 6 & 7 & \begin{array}{l}
\text { Influence tous les aspects } \\
\text { de ma vie }
\end{array}
\end{array}
$$

Une manière différente de mesurer le style explicatif est d'utiliser une méthode d'analyse de contenu à partir d'un matériel écrit ou oral faisant état d'explications causales pour des événement quelconques. Cette technique - dénommée analyse de contenu des explications textuelles (Content Analysis of Verbatim Explanations ou CAVE ; Peterson, Schulman, Castellon \& Seligman, 1992) ou Analyse du Contenu des Explications Spontanées (ACES ; Seligman, 1994) - comprend deux étapes : extraire les événements rapportés par l'individu et ses attributions les concernant, et repérer ce qui relève du locus de causalité, de la stabilité et de la globalité. Différentes conditions doivent être respectées pour utiliser cette technique : (1) les utilisateurs doivent être bien formés, ne pas connaître l'auteur des propos ni l'issue des événements rapportés ; (2) un minimum de cinq événements négatifs est nécessaire 
afin d'identifier le style d'une personne ; (3) il ne peut être question de style que si la personne présente une faible variation dans sa manière d'expliquer les différents événements auxquels elle a été confrontée ; et (4) il est important que la cause relevée soit bien invoquée par la personne dont on cherche à identifier le style (par exemple, si un joueur de football dit dans une interview consécutive à une défaite : « l'entraîneur m'a dit que je n'étais pas en forme en ce moment », l'attribution rapportée dans ces propos semble davantage correspondre à ce que pense l'entraîneur que le joueur ; elle ne pourrait donc pas être retenue dans l'analyse).

Lorsque les événements auxquels la personne a été confrontée ont été repérés, et que ses explications ont été identifiées et catégorisées en termes de locus de causalité, de stabilité et de globalité, une dernière étape consiste à donner un score sur une échelle de 1 à 7 , pour chaque dimension. Le tableau 2 donne un exemple de la manière d'utiliser la technique ACES.

\section{TABLEAU 2}

Exemple d'analyse de contenu d'explications verbales

Les chercheurs examinent minutieusement le contenu de documents écrits ou audio phoniques qui mentionnent des événements accompagnés d'explications causales. Ces faits et ces causes sont ensuite extraits pour être présentés à un jury chargé d'établir un score sur une échelle de un à sept pour chacune des causes selon les dimensions interne versus externe, stable versus instable et global versus spécifique. L'exemple suivant est destiné à illustrer cette méthodologie.

Cela avait vraiment été une mauvaise saison. L'année dernière j'ai manqué la finale des championnats de France d'aviron. J'avais voulu partir vite. Il y avait beaucoup de vent défavorable et j'avais pris des réglages beaucoup trop durs. Je pense que c'est de ma faute parce que je n'avais pas vérifié mon matériel. Je ne fais jamais attention au matériel. J'ai regretté parce que j'aurais pu réaliser une bonne performance.

Fait négatif : j'ai manqué la finale des championnats de France d'aviron... $j$ 'avais pris des réglages beaucoup trop durs

Cause : je n'avais pas vérifié mon matériel. Je ne fais jamais attention au matériel

Score :

Internalité : 7 (je)

Stabilité : 6 (jamais)

Globalité : 2 (le matériel) 
À ce jour, cette méthode a été utilisée à partir de retranscriptions de psychothérapies, d'interviews, de questionnaires ouverts, de discours politiques, de récits sportifs etc. (voir Peterson \& Park, 1998, pour une synthèse de ces travaux). Son principal intérêt est de permettre d'effectuer des études rétrospectives. Si le chercheur dispose de matériel verbal, sur des événements antérieurs de la vie des individus dont le sort à long terme est connu, alors la technique ACES permet de faire très rapidement des études sur plusieurs décennies. Par exemple, Peterson, Seligman et Vaillant (1988) ont utilisé la technique pour prédire avec succès la santé physique de participants qui avaient complété plus de 30 ans plus tôt, un questionnaire analysable par la technique CAVE.

\section{Style explicatif optimiste versus pessimiste}

Avec la naissance du concept de style explicatif, certains auteurs (e.g., Buchanan \& Seligman, 1995 ; Peterson, 1991 ; Seligman, 1991) ont commencé à utiliser les termes " optimisme » et « pessimisme » pour qualifier certains styles explicatifs. Un « style explicatif » ne signifie pas forcément grand chose pour la plupart des gens, mais une vision « optimiste » ou " pessimiste » des causes des événements a probablement plus de sens (Peterson \& Park, 1998). Comme le présente le tableau 3, inspiré de Seligman (1994), une personne qui attribue - de manière récurrente - ses échecs à un facteur considéré comme interne

TABLEAU 3

Définition de l'optimisme et du pessimisme en fonction des explications apportées aux événements positifs et négatifs (d’après Seligman, 1994)

\begin{tabular}{|l|c|c|c|c|}
\hline & \multicolumn{4}{|c|}{ Styles explicatifs } \\
\hline & \multicolumn{2}{|c|}{ Pessimiste } & \multicolumn{2}{c|}{ Optimiste } \\
\hline $\begin{array}{l}\text { Locus } \\
\text { de causalité }\end{array}$ & $\begin{array}{c}\text { Interne } \\
\text { (personnalisa- } \\
\text { tion) }\end{array}$ & $\begin{array}{c}\text { Externe } \\
\text { (extériorisa- } \\
\text { tion) }\end{array}$ & $\begin{array}{c}\text { Externe } \\
\text { (extériorisa- } \\
\text { tion) }\end{array}$ & $\begin{array}{c}\text { Interne } \\
\text { (personnalisa- } \\
\text { tion) }\end{array}$ \\
\hline Stabilité & $\begin{array}{c}\text { Stable } \\
\text { (permanence) }\end{array}$ & $\begin{array}{c}\text { Instable } \\
\text { (transitoire) }\end{array}$ & $\begin{array}{c}\text { Instable } \\
\text { (transitoire) }\end{array}$ & $\begin{array}{c}\text { Stable } \\
\text { (permanence) }\end{array}$ \\
\hline Globalité & $\begin{array}{c}\text { Global } \\
\text { (général) }\end{array}$ & $\begin{array}{c}\text { Spécifique } \\
\text { (particulier) }\end{array}$ & $\begin{array}{c}\text { Spécifique } \\
\text { (particulier) }\end{array}$ & $\begin{array}{c}\text { Global } \\
\text { (général) }\end{array}$ \\
\hline
\end{tabular}


(«c'est de ma faute »), stable (« il n'y a aucune raison que cela change »), et global ("c'est pareil dans tout ce que j'entreprends »), et/ou ses succès à un facteur considéré comme externe («je n'y suis pour rien »), instable («j'ai eu de la chance aujourd'hui »), et spécifique («c'est bien la première fois »), est qualifié de pessimiste. À l'inverse, celui qui attribue ses échecs à un facteur externe, instable, et spécifique, et/ou ses succès à un facteur interne, stable et global, est qualifié d'optimiste.

Partant de ce postulat, les différentes recherches empiriques utilisent généralement la somme des réponses aux scénarii négatifs de l'ASQ (ou de ses dérivés) comme indicateur d'optimisme/pessimisme. En d'autres termes, ils font la somme (ou la moyenne) des réponses des participants aux dimensions causales stabilité, globalité et internalité (mais cette dernière dimension tend à être abandonnée aujourd'hui ; note 1), pour les événements négatifs. Cet indice - dénommé « composite négatif » $(\mathrm{CN})$ - se justifie dans la mesure où les réponses aux échelles globalité et stabilité (et dans une moindre mesure à l'échelle internalité) se révèlent généralement assez corrélées positivement. Plus le score de cet indice est élevé, plus le participant est considéré comme ayant un style pessimiste. À l'inverse, plus le score est faible, plus le participant est considéré comme ayant un style optimiste.

Généralement, les chercheurs calculent aussi un indice à partir des scores obtenus aux dimensions causales, pour les événements positifs. Ce dernier - dénommé « composite positif » $(\mathrm{CP})$ - consiste à faire la somme des dimensions causales stabilité et globalité (et parfois internalité) ; un score élevé reflète une personnalité optimiste. Si l'indice $\mathrm{CN}$ est très utilisé dans les recherches, l'indice $\mathrm{CP}$ a beaucoup plus rarement retenu l'attention des chercheurs (e.g., Taylor \& Brown, 1988), probablement parce qu'il n'est pas possible de tirer autant d'informations des événements positifs que des négatifs, les individus étant moins "soucieux» lorsqu'ils pensent aux premiers, comparativement aux seconds (Langer, 1989).

Conformément aux recommandations de certains auteurs (e.g., Peterson, 1991, 2000), un dernier indice est souvent utilisé par les chercheurs. Dénommé "composite positif moins composite négatif » $(\mathrm{CPMCN})$, il consiste à prendre en compte à la fois les événements positifs, et les événements négatifs, en soustrayant les scores des seconds aux scores des premiers. Plus le score est positif, plus le style de l'individu est optimiste, car il obtient un score élevé à l'indice CP et un score faible à l'indice CN. À l'inverse, plus le score est négatif, plus le style de 
l'individu est pessimiste, car il obtient un score faible à l'indice $\mathrm{CP}$ et un score élevé à l'indice $\mathrm{CN}$.

Pour conclure sur ce point, il semble important de retenir qu'en l'état actuel des recherches, la définition de l'optimisme et du pessimisme dans le cadre de la théorie des styles explicatifs n'est pas consensuelle et reste ouverte. Certains chercheurs considèrent cette caractéristique personnelle uniquement à partir des explications apportées aux événements négatifs ; une majorité des études s'appuie sur les explications présentées par les individus, à la fois aux événements positifs et négatifs ; enfin, une infime minorité des études prend en compte uniquement les événements positifs. Dans le même ordre d'idée, certains chercheurs (e.g., Carver, 1989) sont assez réticents vis-à-vis de l'utilisation des scores composites, qui « diluent » l'information, et recommandent d'utiliser les scores pour chaque dimension causale prise séparément. Par ailleurs, Peterson et Park (1998) invitent à la prudence dans l'utilisation des termes « optimiste » et " pessimiste » dans la mesure où les scores extrêmes sont relativement rares, la majorité des individus obtenant des scores moyens. Ainsi, il ne faudrait pas faire l'amalgame entre un vocable destiné à décrire une manière particulièrement stable d'expliquer les événements, et une typologie de personnalité. Par ailleurs, il existe d'autres manières plus directes de conceptualiser et de mesurer l'optimisme et le pessimisme (pour une synthèse voir Chang, 2000).

Parallèlement à l'avancée des travaux sur la mesure des styles explicatifs, des recherches se sont intéressées aux conséquences de cette variable, tandis que d'autres ont appréhendé ses antécédents. Nous allons d'abord présenter les études qui ont porté sur les conséquences du style, en insistant plus particulièrement sur celles réalisées dans le domaine sportif. Nous développerons ensuite les tendances récentes de la recherche qui tentent d'éprouver l'hypothèse selon laquelle le style explicatif n'est qu'une variable "distale », ou un "facteur de risque » des symptômes de résignation. Enfin, nous présenterons les travaux ayant traité des antécédents du style.

\section{Les conséquences du style}

Un domaine de recherche ayant pour objet l'étude des conséquences du style explicatif sur différentes variables cognitives, motivationnelles, émotionnelles, ou comportementales s'est progressivement développé. 
Il a même tendance aujourd'hui à s'émanciper des problématiques initiales sur la résignation apprise. Ainsi, de nombreuses études ont été réalisées dans lesquelles le style a été considéré comme un corrélat de nombreuses manifestations de la résignation apprise comme la dépression, la maladie, ou l'échec (voir Figure 1). De manière systématique, les personnes au style pessimiste manifestent davantage de symptômes de la résignation apprise par rapport à celles au style optimiste (voir Peterson \& Park, 1998, pour une synthèse). Par exemple, un style pessimiste est lié à de plus faibles performances scolaires (e.g., Nolen-Hoeksema, Girgus \& Seligman, 1986 ; Peterson \& Barrett, 1987) ou professionnelles (e.g., Schulman, 1995), à une moins bonne santé physique ou mentale (e.g., Peterson \& Bossio, 2000), et à davantage de symptômes dépressifs (e.g., Gillham, Shatté, Reivich \& Seligman, 2000). Plus généralement, le style explicatif est associé à des variables comme l'efficience du système immunitaire (Brennan \& Charnetsky, 2000), les blessures (Peterson, Bishop, Fletcher, Kaplan, Yesko, Moon, Smith, Michaels \& Michaels, 2001), les pensées irrationnelles (Ziegler \& Hawley, 2001), la satisfaction conjugale (e.g., Fincham, 2000), la victoire politique (e.g., Zullow, 1995), et différents types d'anxiétés (e.g., Mineka, Pury \& Luten, 1995).

Dans le domaine du sport, il n'existe à notre connaissance que cinq études dont les résultats ont été publiés. Dans une étude conduite avec 50 jeunes joueurs de tennis de haut niveau, Prapavessis et Carron (1988) ont mis en évidence que les joueurs qui présentaient des schémas non adaptatifs sur les plans cognitifs, motivationnels, émotionnels, et comportementaux (mesurés avec des questionnaires) avaient tendance à donner des explications internes, stables et globales à leurs mauvaises performances. Les résultats montrent qu'ils étaient également perçus par leur entraîneur comme étant moins persistants dans leurs matches. Néanmoins, cette étude présente un certain nombre de limites: (1) le style explicatif n'a pas vraiment été mesuré en tant que tel, c'est plutôt la manière dont les joueurs ont expliqué leur récente défaite qui a été prise en compte (Biddle et al., 2001), (2) la mesure du comportement du joueur a été faite de manière indirecte à partir de mesures auto-rapportées ou à partir du jugement des entraîneurs, et (3) style et comportements ont été mesurés au même moment, ce qui rend impossible les inférences de causalité : le style explicatif peut autant causer les déficits constatés que l'inverse.

Deux autres études utilisant la technique ACES pour mesurer le style des joueurs, sont rapportées par Rettew et Reivich (1995). La première a utilisé des équipes de base-ball et la seconde, des équipes de bas- 
ket-ball. Dans les deux cas, les équipes au style optimiste remportaient plus de matches durant la saison étudiée et « rebondissaient » davantage après une défaite que celles au style pessimiste. Toutefois, l'évaluation indirecte du style par la technique ACES, ainsi que la nature rétrospective des données, ne permet pas de prouver le lien de cause à effet entre le style et la performance sportive.

Deux autres études réalisées par Seligman, Nolen-Hoeksema, Thornton et Thornton (1990), avec des nageurs de haut niveau, ont soutenu davantage l'influence du style sur les performances sportives. Dans la première étude, de nature prospective, le style explicatif mesuré en début de saison (l'indice CPMCN) prédisait négativement le pourcentage de compétitions dans lesquelles l'athlète réalisait une contre performance. Dans la seconde étude, l'entraîneur délivrait lors d'une séance test, un feed-back d'échec crédible - à savoir, il signalait une contre performance (allant de 1 seconde 5 à 5 secondes selon les distances de course). Dans un second test réalisé après un repos de 30 minutes, la performance des nageurs au style le plus pessimiste s'est détériorée, ce qui ne fut pas le cas pour les nageurs au style le plus optimiste.

Qu'elles soient conduites dans le domaine du sport, ou dans tout autre domaine, les études sur les styles explicatifs n'ont jamais véritablement testé pleinement les hypothèses de la reformulation de la théorie de la résignation apprise, mais uniquement certains aspects de celleci. En effet, comme le fait remarquer régulièrement Peterson (e.g., Peterson \& Park, 1998 ; Peterson \& Steen, 2002), d'un point de vue théorique, le style explicatif n'est pas considéré comme la cause immédiate et inévitable des problèmes, mais plutôt comme une variable distale (e.g., Peterson \& Steen, 2002) ou un facteur dispositionnel de risque (Peterson \& Park, 1998). Pourtant la plupart des études réalisées dans ce cadre n'ont appréhendé que les corrélats du style, en examinant les liens que cette variable entretient avec d'autres comme la dépression, la maladie ou l'échec ( $c f$. infra).

Il y a deux manières de concevoir et de tester le caractère " distal » d'une variable. La première consiste à envisager l'existence de médiateurs (mediator) entre le style et certains symptômes de résignation. Dans ce cas la variable distale " est à l'origine du déclenchement de l'action d'un médiateur ou de son intensité, qui lui-même influence la réponse " (Rascle \& Irachabal, 2001, p. 99 ; voir également Baron \& Kenny, 1986 ; Brauer, 2000). Parmi les médiateurs possibles, la théorie insiste sur le rôle des attentes (ou expectations) généralisées d'indépendance action 
— résultat (e.g., Peterson \& Steen, 2002). Pourtant, à notre connaissance, il n'existe qu'une seule étude qui ait testé véritablement cette hypothèse (Peterson \& Vaidya, 2001). Dans cette étude, 155 étudiants ont complété un questionnaire mesurant (1) leur style explicatif, (2) leur expectations générales d'occurrence d'événements heureux ou malheureux (e.g., " par rapport aux autres étudiants, quelles sont vos chances de connâ̂tre l'amour véritable cette année »), et (3) la présence ou non de différents symptômes dépressifs. Des analyses par équations structurelles ont corroboré l'existence d'un médiateur — les expectations générales - entre le style et les symptômes de la résignation : un style pessimiste prédisait les expectations d'occurrence d'événements négatifs, qui prédisaient en retour les symptômes de la dépression. Néanmoins, la nature synchronique des données (toutes les variables ont été mesurées au même moment) ne permet pas de confirmer avec certitude la cascade « causale » des événements. Il est, en effet, tout à fait possible d'envisager des influences réciproques (les symptômes dépressifs prédisent les attentes et les styles).

La deuxième manière de concevoir le statut de "variable distale » ou de « facteur de risque » du style explicatif, est de l'envisager comme un modulateur (moderator). Un modulateur est une variable de nature qualitative ou quantitative qui affecte la direction ou l'intensité de la relation entre une variable indépendante et une variable dépendante (voir Baron \& Kenny, 1986 ; Brauer, 2000 ; Rascle \& Iraschabal, 2001). Alors qu'une variable médiatrice nous renseigne sur comment et pourquoi un certain effet a lieu, une variable modulatrice explique quand et sous quelles conditions cet effet se produit (Brauer, 2000). À notre connaissance, il n'existe qu'une seule étude qui ait testé le caractère modulateur du style explicatif (Jackson, Sellers \& Peterson, 2002). Les auteurs de cette étude prédisaient que le style modulait l'influence du stress sur certaines variables négatives comme la maladie ou la détresse psychologique. Les résultats ont effectivement confirmé cette hypothèse. Après avoir mesuré le style explicatif, le stress et la santé perçus de 198 étudiants, Jackson et al. ont demandé aux participants de compléter neuf semaines plus tard, un questionnaire évaluant la présence ou non de maladies. Une interaction significative stress $\times$ style est ressortie : le stress perçu prédisait l'occurrence de maladies pour les individus pessimistes $(\beta=.28, p<.005)$, mais pas pour les individus optimistes $(\beta=-.07, n s)$, et ce, même après avoir contrôlé le niveau initial de santé. Autrement dit, un style pessimiste accentue les effets néfastes du stress 
sur la maladie, alors qu'un style optimiste « immunise » l'individu de ces conséquences néfastes.

Dans le domaine du sport, seules deux études ont - à notre connaissance - testé véritablement le caractère distal du style. L'objectif de la première étude (Martin-Krumm \& Sarrazin, 2002 ; MartinKrumm, Sarrazin, Peterson \& Famose, in press) était tout d'abord de reproduire et d'étendre les résultats de Seligman et al. (1990) sur la capacité de rebond des sportifs après un feed-back d'échec, auprès d'une population de collégien, dans une tâche de basket-ball, et en contrôlant plusieurs variables susceptibles d'influencer la performance sportive comme l'habileté initiale, le sexe, l'habileté perçue et l'importance de réussir. Le deuxième objectif principal de cette étude était d'examiner les processus médiateurs susceptibles d'expliquer comment le style explicatif influence la performance sportive après un échec. Parmi ceuxci, les attentes de réussite et l'état d'anxiété ont été appréhendés. Il était présumé qu'un style pessimiste soit associé à une diminution des attentes de réussite et à une élévation de l'anxiété après un feed-back d'échec, ce qui en retour était susceptible d'entraîner une diminution de la performance à un test ultérieur. Par contraste, il était prévu qu'un style optimiste soit associé à une moindre diminution des attentes de réussite et à une moindre élévation de l'anxiété après échec, ce qui en retour était susceptible de faciliter un « rebond » dans les performances. L'ÉSMSE a été administrée à 278 élèves de collège, et leur score à l'indice $\mathrm{CPMCN}$ a été utilisé pour constituer un groupe de participants au style le plus optimiste et un groupe de participants au style le plus pessimiste. Dans un premier temps, différentes variables de contrôle (e.g., habileté perçue en basket et importance de réussir) ainsi que les attentes des participants quant à leur capacité à réussir un parcours en basket ont été mesurées. Après avoir réalisé ce parcours, un feed-back d'échec a été délivré à l'ensemble des participants. Après une période de repos, les attentes de réussite et l'anxiété somatique (opérationnalisée comme l'augmentation de la fréquence cardiaque par rapport au niveau de base) ont été évaluées, et les participants effectuaient à nouveau le parcours chronométré. Dans ce qu'ils ont d'essentiel, les résultats montrent que les pessimistes ont connu (1) une forte baisse dans leurs attentes de réussite entre les deux tests, (2) une anxiété plus importante à l'approche du deuxième test, et (3) une stagnation de leur performance. À l'inverse, les optimistes ont montré une moindre baisse dans leurs attentes de réussite, une moindre élévation de leur fréquence cardiaque, et une augmentation sensible de leurs performances au second test. L'analyse des pro- 


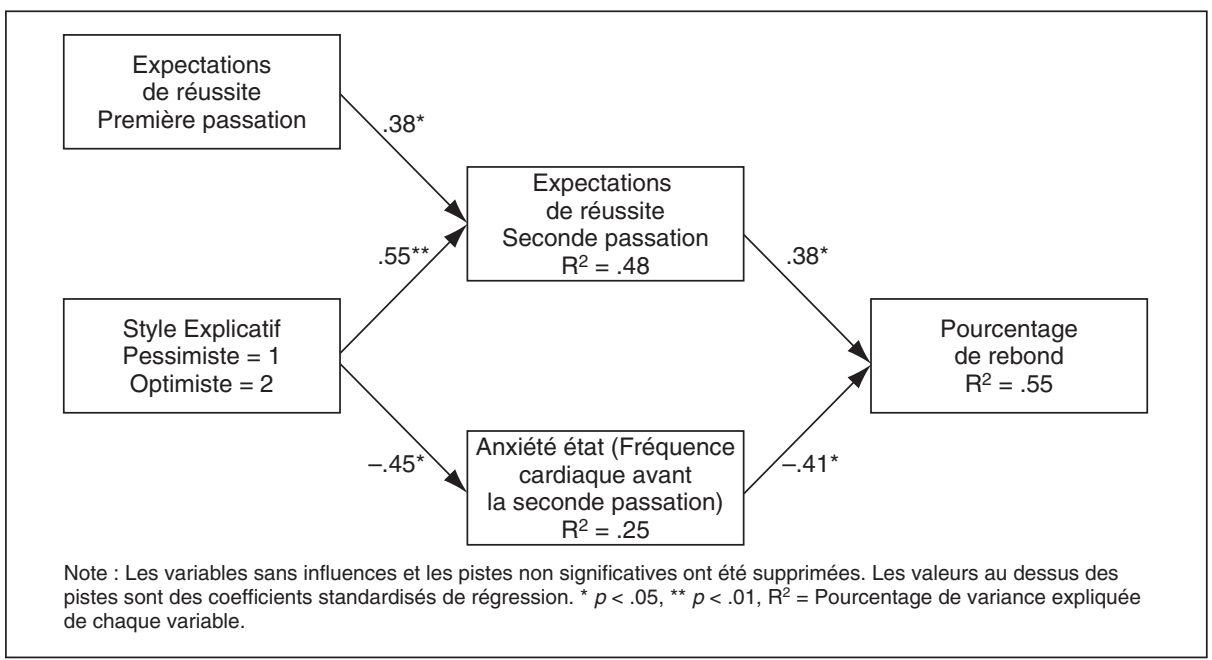

\section{FIGURE 2}

Processus par l'intermédiaire desquels le style explicatif influence le « rebond » dans les performances après un feed-back d'échec (Martin-Krumm, 2002)

cessus - effectuée conformément à la méthode préconisée par Judd et Kenny (1981) — confirme le rôle médiateur des attentes de réussite et de l'anxiété somatique, entre le style explicatif et la faculté de rebond après échec. Les résultats sont visibles sur la figure 2.

Une deuxième étude réalisée en Éducation Physique et Sportive (EPS) (Martin-Krumm, 2002) était destinée à éprouver le caractère modulateur du style, sur une variable qui intervient fortement dans la réussite à l'école: la compétence perçue (Pajares \& Schunk, 2001). D'autre part, comme dans l'étude précédente, les auteurs ont testé l'existence de variables médiatrices entre le style et la réussite en EPS. Conformément au modèle expectation-valeur d'Eccles et Wigfield (2002 ; voir aussi Fontayne \& Sarrazin, 2001), les attentes de réussite et la valeur de la discipline constituent les variables les plus proximales responsables de la réussite dans un domaine d'accomplissement. Les auteurs ont donc émis l'hypothèse selon laquelle le style explicatif interagissait avec la compétence perçue pour prédire à la fois les attentes de réussite et la valeur de l'EPS. En retour, ces deux dernières variables étaient censées prédire la note obtenue par l'élève dans cette discipline.

Pour tester ces hypothèses, 182 élèves de collèges ont été suivis sur une année scolaire. Au début de celle-ci, le style explicatif et la compétence perçue en EPS ont été mesurés. Un mois plus tard, les participants 
ont rempli un second questionnaire appréciant leurs attentes de réussite en EPS et la valeur de cette discipline. Enfin, la moyenne annuelle des élèves a été relevée au terme de l'année scolaire. Les résultats ont, en premier lieu, confirmé le caractère modulateur du style explicatif : une analyse de régression hiérarchique modérée a fait ressortir une interaction significative " compétence perçue $\times$ style explicatif » à la fois sur les attentes de réussite et sur la valeur de l'EPS. Comme le montre la figure 3, pour les pessimistes, la compétence perçue a eu un impact significatif sur la valeur de l'EPS $(b=.48, p<.001)$ : plus elle est faible, plus la valeur de la discipline est faible. Par contraste, pour les optimistes, la compétence perçue n'a pas d'impact significatif sur la valeur de $l^{\prime}$ EPS $(b=.007, n s)$ : une faible compétence perçue n'est pas associée à une dévalorisation particulière de $\mathrm{l}^{\prime} \mathrm{EPS}$. Il est donc vraisemblable que la spécificité des causes évoquées par les individus optimistes pour expliquer un échec n'affecte pas leur estime de soi (Seligman, 1991, 1994). Par conséquent, ceux-ci n'éprouveraient pas le besoin de développer des stratégies de protection de l'estime de soi, telles que la dévalorisation de l'activité (e.g., Harter, 1990). Concernant les attentes de réussite, les résultats montrent que la compétence perçue prédit d'autant plus fortement les attentes de réussite que le style explicatif de l'élève est optimiste ( $b=.73$ vs .59 , respectivement pour les plus optimistes et les plus pessimistes; cf. Figure 3). Autrement dit, si le style

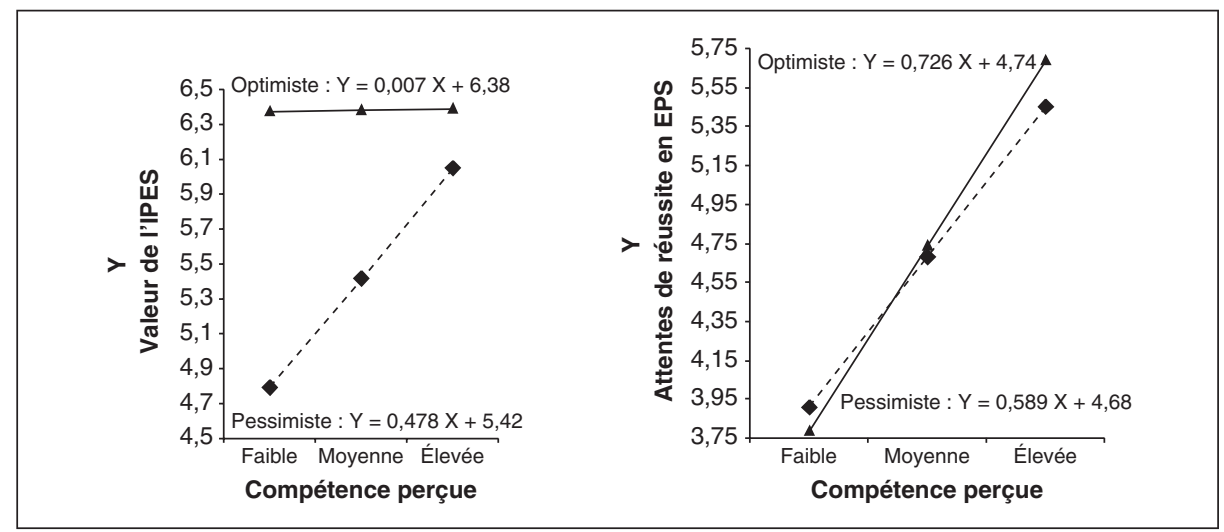

FIGURE 3

Rôle modulateur du style explicatif dans l'influence de la compétence perçue sur la valeur de l'EPS (graphique de gauche) et les attentes de réussite en EPS (graphique de droite), d'après Martin-Krumm (2002) 


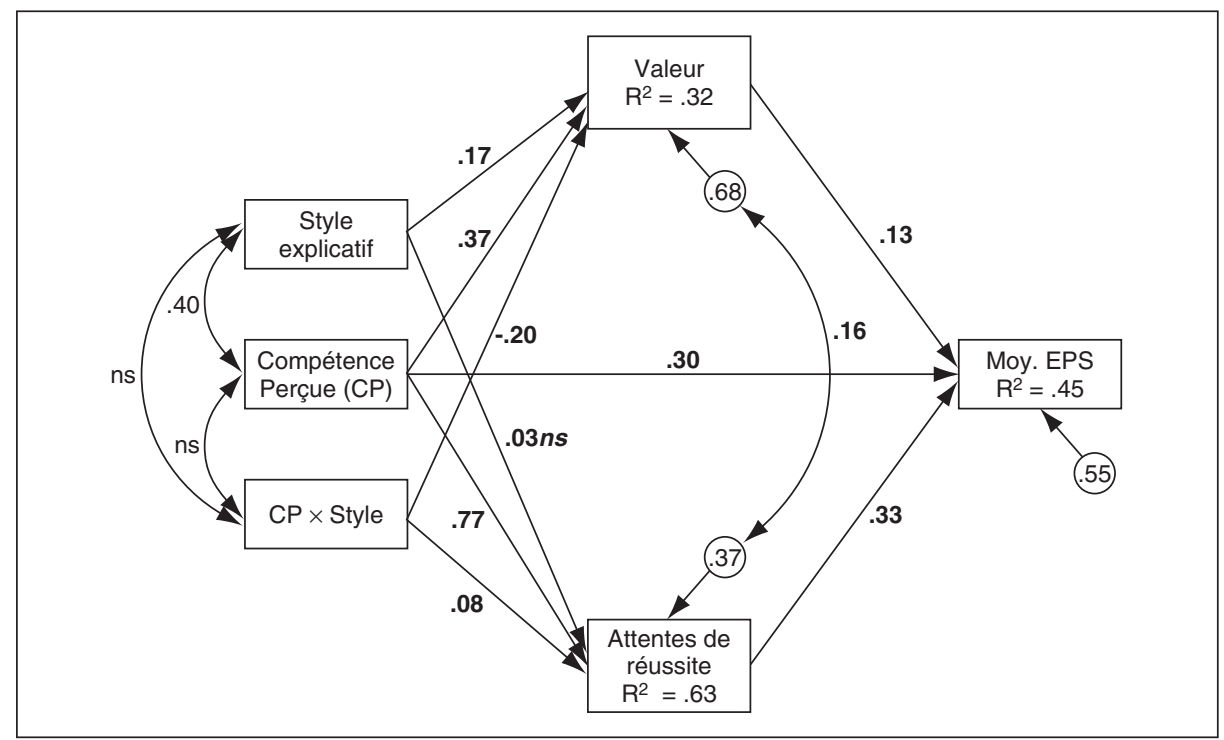

\section{FIGURE 4}

Modèle structurel des relations entre le style explicatif, la compétence perçue, les attentes de réussite et la valeur pour prédire les notes en EPS, d'après Martin-Krumm (2002). Les paramètres sont standardisés, et significatifs à $p<.05$. Les résidus sont présentées dans les petits cercles.

Note : Les variables sans influences et les pistes non significatives ont été supprimées. Les valeurs au dessus des pistes sont des coefficients standardisés de régression. $* p<.05$, ** $p<.0 \mathrm{I}, \mathrm{R}^{2}=$ Pourcentage de variance expliquée de chaque variable.

n'exerce aucune influence quand la compétence perçue est faible ou moyenne, les conséquences d'une compétence perçue élevée sur les attentes de réussite sont d'autant plus fortes que l'élève est optimiste.

Enfin, les résultats de cette étude confirment le caractère distal du style explicatif, sur la réussite en EPS. Des analyses par équations structurelles montrent que quand les attentes de réussite et la valeur de 1'EPS sont contrôlées, le style explicatif n'a plus d'effet sur la note (cf. Figure 4). Autrement dit, l'influence du style explicatif sur la note s'exerce par l'intermédiaire de variables plus proximales présumées par Eccles (e.g., Eccles \& Wigfield, 2002) : les attentes de réussite et la valeur de l'activité. 


\section{Antécédents du Style Explicatif}

Après avoir confirmé l'influence directe ou indirecte du style sur la performance sportive, il semble important à présent de s'interroger sur l'origine de cette caractéristique personnelle. D'où provient cette manière relativement stable que nous avons d'expliquer les événements auxquels nous sommes confrontés ? Le style est-il stable ou susceptible d'évoluer dans le temps ? Les études qui se sont intéressées à ces questions n'ont pas eu la performance sportive comme contexte de référence. Toutefois, compte tenu de la généralité relative du style explicatif, nous allons présenter rapidement les principaux résultats. Il est possible de regrouper les antécédents du style en trois grandes catégories : les facteurs génétiques, les expériences singulières, et les facteurs sociaux.

Selon Buchanan et Seligman (1995), il est possible qu'il puisse y avoir une composante génétique dans la genèse du style explicatif. Une corrélation de l'ordre de .48 a été observée entre les styles explicatifs des jumeaux monozygotes, alors que le lien n'est pas significatif pour des jumeaux dizygotes (Schulman, Keith \& Seligman, 1991). Toutefois, il semble plus probable que les facteurs génétiques n'aient qu'une influence indirecte sur le style explicatif (Peterson \& Park, 1998), en prédisposant l'individu à vivre certaines expériences positives ou négatives. Les qualités physiques, psychologiques, morphologiques etc., déterminées par des facteurs génétiques, peuvent amener les individus à faire certaines expériences heureuses ou malheureuses, qui influenceront en retour leur style.

Quelles sont les conséquences d'un traumatisme important sur le style explicatif des individus? Les études montrent que les expériences singulières telles les maladies, les traumas, les négligences ou les échecs cuisants dans des domaines importants, peuvent avoir des conséquences sensibles sur la genèse d'un style explicatif pessimiste (e.g., Brewin \& Furnham, 1986 ; Nolen-Hoeksema, Girgus \& Seligman, 1991).

Enfin, les facteurs sociaux comme les autrui significatifs (i.e., les personnes importantes pour l'individu comme ses parents, son/sa meilleur(e) ami(e), les enseignants etc.) ou les médias semblent contribuer à forger un style explicatif particulier. Selon Peterson et Park (1998) les feed-back des parents au regard des comportements de leur(s) enfant(s) modèleraient de manière durable sa manière d'expliquer et d'interpréter les événements auxquels il est confronté tout au long de sa vie. Par exemple, Radke-Yarrow, Belmont, Nottelman, et Bottomly 
(1990) ont trouvé une forte association entre le nombre de commentaires désapprobateurs de la mère à l'égard de son enfant, et le nombre d'allusions négatives à soi-même de l'enfant. D'autres études soulignent le rôle des enseignants dans la stabilisation du style explicatif de l'enfant. Par exemple Heyman, Dweck, et Cain (1992), ont montré que les critiques des enseignants pouvaient, dès l'école maternelle, influencer les perceptions négatives que les enfants avaient d'eux-mêmes. Un autre type d'influence concerne l'impact de la confiance que les autrui significatifs accordent à l'enfant. Selon Eisner (1995), un manque de confiance de leur part peut entraîner le développement d'un style pessimiste, alors que leur confiance entraînerait plutôt un style optimiste. Enfin, certains travaux se sont penchés sur l'influence des médias télévisés (e.g., Hearn, 1991). Les scènes répétées de violences dans les fictions ou les journaux télévisés, exacerberaient un sentiment d'insécurité susceptible de générer le pessimisme (e.g., Hearn, 1991; NolenHoeksema, 1987); ceci d'autant plus quand les événements violents sont présentés comme durables et omniprésents, et que les téléspectateurs sont jeunes (Peterson \& Steen, 2002).

En résumé, la question des antécédents du style amène des réponses multiples. Le style semble être le résultat de facteurs biologiques, psychologiques et sociaux, chacun contribuant vraisemblablement à cristalliser en l'individu une manière de percevoir le monde qui l'entoure, sans qu'il soit possible à l'heure actuelle de préciser le rôle et la nature de chacun d'entre eux. D'autre part, il est probable qu'un processus complexe s'engage dans lequel les événements influencent autant la construction du style, que ce dernier n'influence les événements (Peterson et al., 1993). Comme la dépression, le style explicatif peut donc être à la fois une cause et une conséquence (Nolen-Hoeksema et al., 1986). Par exemple, un style explicatif pessimiste maximise la probabilité d'occurrence d'événements négatifs, qui en retour renforcent le style pessimiste de l'individu.

Le style évolue-t-il en fonction de l'âge ? Y-a-t-il des différences en fonction du sexe ? Il semblerait que oui. Selon Nolen-Hoeksema et Girgus (1995), le style semble se cristalliser vers l'âge de 9 ans, mais certains événements peuvent encore le faire évoluer jusqu'à 13-14 ans. Ces auteurs ont conduit une étude longitudinale sur 5 ans, dans laquelle les enfants remplissaient tous les six mois un questionnaire permettant d'identifier leur style explicatif. Les résultats ont révélé qu'entre 9 et 13 ans, les attributions relatives aux événements positifs devenaient plus stables et globales (donc correspondant à un style de plus en plus 
optimiste), alors que celles pour les événements négatifs n'évoluaient pas. D'autre part, le style explicatif des garçons semble évoluer différemment de celui des filles. Les premiers deviennent de plus en plus optimistes après 13 ans, alors que les filles deviennent de plus en plus pessimistes entre 11 et 13 ans, et n'évoluent plus par la suite. L'existence d'un style plus pessimiste (ou moins optimiste) chez les filles a été confirmée dans les domaines sportif (Seligman et al., 1990), ou scolaire (e.g., Nolen-Hoeksema, 1987).

Plusieurs hypothèses ont été émises pour expliquer ces différences. Les biais évaluatifs dont sont victimes les filles (e.g., Basow, 1986) ont été mis en cause, en particulier l'interprétation de leurs succès et échecs par les autrui significatifs. Par exemple, les succès sont davantage expliqués en termes de causes internes et stables (e.g., l'aptitude) pour les garçons, et en termes de causes instables (e.g., l'effort, l'aide de l'entourage) pour les filles. À l'inverse, les échecs sont davantage expliqués en termes de causes instables (e.g., le manque de travail) pour les garçons, et en termes de causes internes et stables (e.g., la manque d'aptitudes) pour les filles (e.g., Dweck, Davidson, Nelson \& Enna, 1978). Dans le même ordre d'idée, l'attitude des parents, qui ont tendance à pousser les garçons et à freiner les filles dans leurs expériences, a aussi été invoquée (e.g., Firth, 1982). Enfin, la manière dont les garçons et les filles perçoivent leurs changements morphologiques à l'adolescence peut aussi expliquer leurs différences au niveau du style explicatif. Si les garçons ont plutôt tendance à apprécier ces changements (le développement morphologique et des caractéristiques sexuelles secondaires, la prise de masse musculaire etc., leur permettant de se rapprocher des «canons » de la virilité), les filles semblent davantage souffrir de la modification de leur silhouette, notamment de l'augmentation des masses graisseuses qui les éloigne des canons en vigueur de la féminité (e.g., Simmons, Blyth, Van Cleave \& Bush, 1979). Cette frustration de ne pas pouvoir contrôler les changements de silhouette qu'elles subissent (en association avec la valeur sociale accordée à la minceur) contribue à renforcer une vision pessimiste des événements, plus fréquente chez les filles (e.g., McCarthy, 1990).

\section{Conclusion et perspectives}

L'objet de cet article était de présenter les différentes avancées que la recherche sur les styles attributionnels ou styles explicatifs a connues. Ce concept tire ses racines de la théorie de la résignation apprise (e.g., 
Seligman \& Maier, 1967 ; Seligman, 1975) qui place au centre du modèle, l'apprentissage d'incontrôlabilité (ou de l'inutilité de ses efforts). Les critiques que cette théorie a suscitées, ont conduit les chercheurs à une reformulation du modèle initial empruntant des éléments à la théorie de l'attribution causale (Abramson et al., 1978). À présent, la manière relativement récurrente d'expliquer - en terme de causes stables ou instables, et globales ou spécifiques - les événements positifs ou négatifs susceptibles d'arriver est présumée constituer une variable intervenant dans les symptômes de résignation. Plus précisément, contrairement à un style optimiste (i.e., la propension à expliquer les échecs en termes de cause instable et spécifique, et les réussites en termes de cause stable et globale), un style pessimiste (i.e., la propension à expliquer les échecs en termes de cause stable et globale, et les réussites en termes de cause instable et spécifique) est présumé conduire à certains symptômes de résignation comme la passivité, l'anxiété, les échecs, la maladie ou des déficits cognitifs. Certains travaux ont confirmé ces résultats dans le domaine sportif (Prapavessis \& Carron, 1988 ; Rettew \& Reivich, 1995 Seligman et al., 1990).

Après avoir été considéré comme un corrélat de différents symptômes, le style est maintenant appréhendé comme une variable distale ou un facteur de risque. Les travaux récents conduits à la fois dans le domaine de la pathologie (e.g., Jackson et al., 2002 ; Peterson \& Steen, 2002) ou dans celui du sport (Martin-Krumm, 2002 ; Martin-Krumm et al., in press) ont confirmé l'effet indirect du style sur différentes variables, comme la performance sportive. Ils montrent comment le style agit sur certains médiateurs qui en retour jouent un rôle sur les résultats obtenus par l'individu. Ils permettent également de décrire l'effet modulateur du style - une variable pouvant immuniser des influences néfastes de certaines variables ou au contraire les aggraver. Néanmoins, certains aspects théoriques, méthodologiques et empiriques méritent encore d'être développés.

\section{Du simple lien de cause à effet entre style et performance, à la recherche d'autres médiateurs}

Le rôle d'autres médiateurs motivationnels, entre le style et certains comportements de résignation reste encore à déterminer. Compte tenu des différents modèles rendant compte du fonctionnement de la motivation humaine dans le domaine du sport (pour une synthèse, voir Cury 
\& Sarrazin, 2001), il semble prometteur d'envisager les buts poursuivis par l'individu comme faisant partie de ces médiateurs potentiels. On peut en effet postuler que la poursuite de buts d'évitement (e.g., comportements de fuite ou de renoncement; voir Cury \& Da Fonséca, 2001), ou l'a-motivation (e.g., absence de motivation ; voir Vallerand \& Grouzet, 2001) soient plus fréquentes parmi les sportifs ayant un style pessimiste. Par ailleurs, d'autres études dans le contexte de la performance sportive sont nécessaires afin d'éprouver également des modèles dans lesquels le style pourrait moduler l'effet de prédicteurs de la performance comme le stress.

\section{Tester les effets de l'environnement social sur le développement du style en contexte sportif}

D'une manière générale, des travaux sur les antécédents des styles explicatifs dans le domaine sportif semblent nécessaires dans la mesure où aucune étude, à notre connaissance, n'a été réalisée dans ce domaine. On peut dont s'interroger sur la cascade des événements qui conduit un sportif à adopter un style pessimiste. Quelles sont les causes possibles?

L'entraîneur, les parents, les équipiers, la confrontation à un climat de compétition dès le plus jeune âge sont-ils des facteurs susceptibles d'influencer l'installation d'un style particulier ? Le rôle des pressions sociales exercées sur les jeunes sportifs, parfois très jeunes, dans le cadre de certaines activités sportives comme la gymnastique ou le tennis mérite d'être abordé et pourrait contribuer à compléter certains modèles permettant d'expliquer les processus impliqués dans l'abandon sportif (e.g., Sarrazin \& Guillet, 2001).

\section{Passer d'un style pessimiste à un style optimiste dans le contexte de la performance sportive}

Les résultats présentés plus haut ont souligné les bienfaits d'un style optimiste et les méfaits d'un style pessimiste pour les individus, en général, et les sportifs en particulier. La carrière de ces derniers étant parsemée de succès, mais aussi de désillusions profondes, la capacité à gérer les échecs et à repartir de l'avant semble constitutive de la personnalité des champions. Mais que faire, lorsqu'un sportif présente un style explicatif pessimiste ? N'est-il pas possible de " rééduquer » un tel style, afin de le transformer en style plus optimiste? 
Il existe plusieurs recherches dont l'objet porte sur la modification du style explicatif. Certaines se sont déroulées dans le cadre de programmes plus vastes destinées à lutter contre la dépression (e.g., Jaycox, Reivich, Gillham \& Seligman, 1994). Une seconde ligne de recherche a été conduite dans le domaine scolaire, avec pour objectif d'apprendre aux élèves à modifier leurs attributions causales. Les résultats ont montré qu'en encourageant les élèves à attribuer les échecs scolaires au manque d'effort (une cause spécifique et instable), ceux-ci étaient plus persistants sur les tâches (e.g., Andrews \& Debus, 1978 ; Fowler \& Peterson, 1981). Cet entraînement à la modification des explications causales semble même plus efficace que le simple fait de placer l'individu en condition de réussite. Par exemple, Aydin (1988) a comparé les effets de deux programmes destinés à un groupe d'élèves impopulaires au style explicatif pessimiste. Ces derniers ont été assignés soit à une condition de réentraînement attributionnel (durant 10 semaines, on leur lisait des scénarii dans lesquelles le rejet social était attribué à un manque d'effort plutôt qu'à un manque d'aptitude, et on leur demandait ensuite de " jouer un rôle » dans l'histoire racontée), soit à une condition de succès (dans laquelle l'expérimentateur manipulait le feed-back d'un prétendu test de compétence sociale, de sorte que l'enfant reçoive $90 \%$ de feedback de réussite à la fin de la période), soit à une condition de contrôle. Les résultats ont révélé que seul l'entraînement à la modification des attributions causales changeait le style explicatif des participants, et améliorait la relation de ces enfants impopulaires à leurs pairs, même s'ils restaient toujours relativement mal perçus.

Il ne faudrait pas conclure trop hâtivement de ces résultats que les succès ne sont pas importants pour modifier un style explicatif pessimiste. Il semble uniquement, qu'à court terme, l'entraînement à la modification des attributions soit plus efficace que simplement donner l'illusion de réussir. Néanmoins, il est fortement probable qu'une modification du style vers davantage d'optimisme, qui ne soit pas assortie de succès subséquents, conduise rapidement à un retour en arrière - à savoir, une manière pessimiste d'expliquer les événements.

De tels programmes, spécifiquement conçus pour le domaine du sport, permettraient d'acquérir des techniques limitant l'impact des événements négatifs, comme les blessures ou les performances décevantes, sur la vie des sportifs. Une approche de cet ordre mériterait une attention particulière et pourrait ouvrir des perspectives de recherche prometteuses dans leurs applications tant dans le domaine du sport que dans celui de l'EPS. 


\section{Différents niveaux de généralité et différents styles explicatifs}

Dans les premières conceptualisations du style explicatif, cette variable se plaçait à un niveau très élevé de généralité. À l'instar de l'ASQ (cf. infra), les outils interrogaient les participants sur les causes d'événements négatifs ou positifs, susceptibles d'arriver dans différentes situations de vie (professionnelle, familiale, amicale etc.). Compte tenu de ce niveau de généralité, il n'est pas surprenant que les individus au style pessimiste soient enclins à la dépression et manifestent des comportements de résignation généralisée. Néanmoins, les profils si extrêmes sont rares ; et il est possible de trouver des comportements de résignation qui soit plus circonscrits à un domaine d'accomplissement particulier, sans pour autant que l'individu manifeste les symptômes pathologiques de la dépression. C'est la raison pour laquelle, plusieurs auteurs recommandent d'évaluer le style explicatif en utilisant des outils spécifiques au contexte (e.g., Cutrona, et al., 1985 ; Peterson \& Park, 1998 ; Peterson, 2000). Ainsi, à l'instar des modélisations contemporaines sur le concept de soi (e.g., Marsh, 1998) ou sur la motivation (e.g., Vallerand, 1997), il semble heuristique d'envisager l'existence de différents styles explicatifs relativement circonscrits à des domaines particuliers comme l'école, le sport, le travail, les relations sociales etc., qui sont susceptibles de se généraliser à un style explicatif plus vaste (e.g., Peterson \& Park, 1998). La validité prédictive de ces différents styles, ainsi que les liens qu'ils entretiennent, mériteraient d'être analysés dans les travaux ultérieurs.

D'autre part, les travaux dont nous avons fait état, ont souvent comparé les styles explicatifs optimiste et pessimiste. Or comme nous l'avons fait remarquer, les scores extrêmes sont relativement rares. La majorité des participants obtiennent des scores bas aux deux échelles d'événements positifs et négatifs, tandis que certains rapportent des scores élèves à ces deux échelles (e.g., Peterson \& Park, 1998). Les travaux futurs devraient ajouter un groupe de participants obtenant des scores faibles aux deux échelles et un autre groupe de participants obtenant des scores élevés, afin de comparer les résultats de ces 4 profils. D'autre part, l'étude des réactions de participants présentant ces différents profils, suite à des feed-back d'échec et de réussite, apporterait également des informations sur le caractère plus ou moins adaptatif de chacun d'eux.

En définitive, si l'objectif est d'expliquer ou de décrire ce qui conduit les uns à voir leurs performances stagner ou baisser après échec, et 
les autres à rebondir, les futures études qui s'intéresseront au style explicatif pourront apporter une aide aux cadres sportifs ou aux enseignants dans leur manière d'intervenir auprès de leurs athlètes ou de leurs élèves. Ce type de recherches permettra aux études réalisées dans le contexte sportif de s'intégrer dans le courant plus général de la Psychologie Positive dont l'objet est de comprendre et de développer les qualités qui conduisent les individus, les communautés et les sociétés à être performants et à s'épanouir. Ce courant de la psychologie a pour objet d'étude l'ensemble des facteurs qui influencent le bien-être, les facultés de rebond, les " états de grâce » (flow) parfois ressentis lors de situations particulières (e.g., Seligman, 2002), comme il est possible d'en vivre dans le domaine du sport de compétition ou de loisir.

\section{Bibliographie}

Abramson, L.Y., Seligman, M.E. P., TeAsDale, J., (1978). Learned helplessness in humans: Critique and reformulation. Journal of Abnormal Psychology, Vol. 87, 1, 49-74.

AYDIN, G. (1988). The remediation of children's helpless explanatory style and related unpopularity. Cognitive and Research, 12, 155-165.

ANDREWS, G.R. \& DEBUS, L.R. (1978). Persistence and the causal perception of failure : modifying cognitive attributions. Journal of Éducational Psychology, 70, 154-166.

BARON, R. \& KENNY, D. (1986). The moderator-mediator variable distinction in social psychological research : Conceptual, strategic, and statistical considerations. Journal of Personality and Social Psychology, 51, 1173-1182.

BASOW, S.A. (1986). Gender stereotypes : Traditions and alternatives ( $2^{\text {nd }}$ ed.). Montery, CA : Brooks/cole.

Biddle, S.J., Hanrahan, S.J. \& Sellars, C.N. (2001). Attributions : Past, present, and future. In R.N. Singer, H.A. HAuSENBlas \& C.M. JANELle (Eds.), Handbook of Sport Psychology (pp. 444-469), Second Edition, John Wiley and Sons.

BRAUER, M. (2000) L'identification des processus médiateurs dans la recherche en psychologie. L'Année Psychologique, 100, 661-681.

BRENNAN, F.X. \& CHARNETSKY, C.J. (2000). Explanatory style and immunoglobulin A (IgA). Integrative Physiological and Behavioral Science, Vol. 35, 4, 251-255.

BREWIN, C.R. \& FURNHAM, A. (1986). Attributional versus preattributional variables in self-esteem and depression : A comparison and test of learned helplessness theory. Journal of Personality and Social Psychology, 50, 1013-1020.

BuCHANAN, G.M. \& SEligman, M.E. P. (1995). Explanatory Style. Hillsdale, NJ : Erlbaum. 
CARVER, C.S. (1989). How should multi-faced personality construct be tested ? Issues illustrated by self-monitoring, attributional style, and hardiness. Journal of Personality and Social Psychology, 56, 577-585.

CHANG, C.E. (2000). Optimism \& Pessimism. Implications for theory, research, and practice. Washington, DC : APA.

CURY, F. \& Da FONSÉCA, D. (2001). Approche/évitement et théorie des buts d'accomplissement: données empiriques et avancées conceptuelles. In F. Cury \& P. Sarrazin (Eds), Théories de la motivation et pratiques sportives : État des recherches (pp. 121-142). Paris : PUF.

CURY, F. \& SARRAZIN, P. (2001). Théories de la motivation et pratiques sportives : État des recherches. Paris : PUF.

Cutrona, C.E., Russel, D. \& JONES, R. (1985). Cross-situational consistency in causal explanations : Does attributional style exist? Journal of Personality and Social Psychology, 47(5), 1043-1058.

DWECK, C., DAVIDSON, W., NELSON, S. \& ENNA, B. (1978). Sex differences in learned helplessness : II. The contingencies of evaluative feed-back in the classroom and III. An experimental analysis. Developmental Psychology, 14, 268-276.

DYKeMA, J., BerGbower, K., DOCTORA, J.D. \& Peterson, C. (1996). An attributional style questionnaire for general use, Journal of Psychoeducational Assessment, 14, 100-108.

ECCLES, J.S. \& WigfieLD, A. (2002). Motivational beliefs, values, and goals. Annual Review of Psychology, 53, 109-132.

EISNER, J.P. (1995). The origins of explanatory style : Trust as a determinant of optimism and pessimism. In G.M. Buchanan \& M.E. P. Seligman, Explanatory Style (pp. 49-55). Hillsdale, NJ : Erlbaum.

FINCHAM, F.D. (2000). Optimism and The Family. In J.E. Gillham (Ed.), The science of optimism and hope, research essays in honor of M.E. P. Seligman (pp. 271-298), Templeton Foundation Press.

FIRTH, M. (1982). Sex discrimination in job opportunities for women. Sex Roles, 8, 891-901.

FONTAYNE, P. \& SARRAZIN, P. (2001). Genre et motivation dans le domaine du sport. In F. CURY, et P. SARRAZIN (Eds.), Théories de la motivation et pratiques sportives : État des recherches (pp. 277-295). Paris : PUF.

FOWLER, W.J. \& PETERSON, L.P. (1981). Increasing reading persistence and altering attributional style of learned helpless children. Journal of Éducational Psycho$\log y, 73,251-260$.

FRANKEL, A. \& SNYDER, M.L. (1978). Poor performance following unsolvable problems : Learned helplessness or egotism? Journal of Personality and Social Psychology, 36, 1415-1423.

Gernigon, C., Fleurance, F. \& Reine, B. (2000). Effects of uncontrollability and failure on the development of learned helplessness in perceptual-motor tasks. Research Quaterly for Exercise and Sport, 71(1), 44-54. 
Gernigon, C. Fleurance, P., WolfF, M. \& Reine, B. (1998). Conditions d'installations et conséquences de la résignation apprise dans les tâches motrices. Science et Motricité, 34, 7-18.

Gillham, J.E., Shatté, A.J., Reivich, K.J. \& Seligman, M.E. P. (2000). Optimism, pessimism, and explanatory style. In E.C. Chang (Ed.), Optimism \& Pessimism. Implications for theory, research, and practice (pp. 53-75). Washington, DC : APA.

HANRAHAN, S. \& GROVE, J.R. (1990). A Short form of the Sport Attributional Style Scale. The Australian Journal of Science and Medicine in Sport, 22 (4), 97-101.

HANRAHAN, S., GROVE, J.R. \& HATTIE, J.A. (1989). Development of a questionnaire measure of sport-related attributional style. International Journal of Sport Psychology, 20, 114-134.

HARTER, S. (1990). Causes, correlates and the functional role of global self-worth : A life span perspective. In J. Kolligian \& R. Stenberg (Eds.), Perceptions of competence and incompetence across a life span (pp. 67-98). New Haven, CT : Yale University Press.

HEARN, G. (1991). Entertainment manna : Does television viewing lead to appetitive helplessness ? Psychological Reports, 68, 1179-1184.

HEYMAN, G.D., DWECK, C.S. \& CAIN, K.M. (1992). Young children's vulnerability to self-blame and helplessness : Relationship to beliefs about goodness. Child Development, 63, 401-415.

Hiroto, D.S. (1974). Locus of control and learned helplessness. Journal of Experimental Psychology, 102, 187-193.

HiRoto, D.S. \& SELIGMAN, M.E. P. (1975). Generality of learned helplessness in man. Journal of Personality and Social Psychology, 31, 311-327.

JACKSON, B., Sellers, R.M. \& Peterson, C. (2002). Pessimistic explanatory style moderates the effect of stress on physical illness. Personality and Individual Differences, 32, 567-573.

JayCox, L.H., Reivich, K., Gillham, J. \& SEligman, M.E. P. (1994). Prevention of depressive symptoms in school children. Behaviour Research and Therapy, 32, 801-816.

JUDD, C.M. \& KENNY, D.A. (1981). Estimating the effects of social interventions. Cambridge, UK : Cambridge University Press.

Kaslow, N.J., Tennenbaum, R.L. \& Seligman, M.E. P. (1978). The KASTAN : A children's attributional style questionnaire. Unpublished manuscript, University of Pennsylvania.

Kelley, H.H. (1973). The process of causal attribution. American Psychologist, 28, 107-128.

LANGER, E.J. (1989). Mindfulness. Reading, MA : Addison-Wesley.

MAIER, S.F. \& SELIGMAN, M.E. P. (1976). Learned helplessness : Theory and evidence. Journal of Experimental Psychology, General, 105, 3-46.

MARSH, H.W. (1998). Age and gender effects in physical self-concepts for adolescent elite athletes and nonathletes: A multicohort-miltioccasion Design. Journal of Sport and Exercise Psychology, 20, 237-259. 
MARTIN-KRUMm, C. (2002). Styles attributionnels et performance motrice. Comment le style attributionnel influence la performance sportive et la réussite en Éducation Physique et Sportive. Thèse de doctorat non publiée, spécialité STAPS, Université Paris XI - Orsay.

MARTIN-KRUMM, C. \& SARRAZIN, P. (2002, june). Explanatory style and ability to rebound after failure : A process analysis in a sport task. Communication presented at the first Positive Psychology Conference, Winchester, U.K.

MARTin-KRUMm, C., SARRAZIN, P., FONTAYNE, P. \& FAMOSE, J.P. (2001). Validation d'une version préliminaire de l'Échelle Sportive de Mesure du Style Attributionnel. Communication présentée au ge congrès de l'ACAPS. Valence, France, novembre 1-3.

MARTin-Krumm, C., SARRAZIN, P., Peterson, C. \& FAMOSE, J.P. (2003). Explanatory style and resilience after sport failure. Personality and Individual Differences, 35, 1685-1695.

McCARTHY, M. (1990). The thin ideal, depression and eating disorders in women. Behavior Research and Therapy, 28, 205-215.

MiKulincer, M. (1994). Human Learned Helplessness : A coping perspective. New York : Plenum Press.

MikUlinCER, M. \& NIZAN, B. (1988). Causal attribution, cognitive inference, and the generalization of learned helplessness. Journal of Personality and Social Psycho$\log y, 55,470-478$.

MilleR, I.W. \& NORMAN, W.H. (1979). Learned helplessness in humans : A review and attribution theory model. Psychological Bulletin, 86, 93-119.

MineKA, S., Pury, C.L. \& LuTEN, A.G. (1995). Explanatory Style in Anxiety and Depression. In G.M. Buchanan \& M.E. P. Seligman (Eds.), Explanatory Style (pp. 135-158), Hillsdale, NJ : Erlbaum.

NOLEN-HoEKSEMA, S. (1987). Sex differences in unipolar depression : Evidence and theory. Psychological Bulletin, 101, 259-282.

Nolen-HoeKsemA, S. \& GiRGUS, J.S. (1995). Explanatory style and achievement, depression, and gender differences in childhood and early adolescence. In G.M. BuchanAN \& M.E. P. SEligman (Eds.), Explanatory Style (pp. 57-70). Hillsdale, NJ : Erlbaum.

Nolen-Hoeksema, S., Girgus, J.S. \& Seligman, M.E. P. (1986). Learned helplessness in children: A longitudinal study of depression, achievement, and explanatory style. Journal of Personality and Social Psychology, 51, 435-442.

Nolen-HoeKsema, S., Girgus, J.S. \& Seligman, M.E. P. (1991). Sex differences in depression and explanatory style in children. Journal of Youth and Adolescence, 20, 233-245.

Overmier, J.B. \& SELIGMAN, M.E. P. (1967). Effects of inescapable shock upon subsequent escape and avoidance learning. Journal of Comparative and Physiological Psychology, 63, 28-33.

PAJARES, F. \& SCHUNK, D.H. (2001). Self-beliefs and school success : Self-efficacy and self- concept in academic settings. In R. Riding \& S. Rayner (Eds.), Self-perception (pp. 239-266). London : Ablex Publishing. 
Peterson, C. (1991). The Meaning and Measurement of Explanatory Style. Psychological Inquiry, Vol. 2, 1, 1-10.

Peterson, C. (2000). The Future of Optimism, The American psychologist, Vol. 55, 1, $44-45$.

PETERSON, C. \& BARRETT, L. (1987). Explanatory style and academic performance among university freshmen. Journal of Personality and Social Psychology, 53, 603607.

Peterson, C., Bishop, M.P., Fletcher, C.W., Kaplan, M.R., Yesko, E.S., MoON, C.H., SMITH, J.S., MichaEls, C.E. \& MichaELS, A.J. (2001). Explanatory style as a risk factor for traumatic mishaps. Cognitive Therapy and Research, 25(6), 633649.

Peterson, C. \& Bossio, L.M. (2000). Optimism and physical well-being. In E.C. Chang (Ed.), Optimism E pessimism. Implications for theory, research, and practice (pp. 127-145). Washington, DC : APA.

Peterson, C., Buchanan, G.M. \& Seligman, M.E. P. (1995). Explanatory Style : History and evolution of the field. In G.M. Buchanan \& M.E. P. Seligman (Eds), Explanatory Style (pp. 1-20). Hillsdale, NJ : Lawrence Erlbaum Associates.

Peterson, C., MAIER, S.F. \& Seligman, M.E. P. (1993). Learned Helplessness, a theory for the age of personal control. New York: Oxford University Press.

Peterson, C. \& PARK, C. (1998). Learned helplessness and explanatory style. In D.F. Barone, V.B. Van Hasselt \& M. HerSen (Eds), Advanced Personality (pp. 287310). New York : Plenum.

Peterson, C., Schulman, P., Castellon, C. \& Seligman, M.E. P. (1992). CAVE : Content Analysis of Verbatim Explanations. In C.P. Smith (Ed), Motivation and personality: Handbook of thematic content analysis (pp. 383-392). New York: Cambridge University Press.

Peterson, C. \& Seligman, M.E. P. (1984). Causal explanations as risk factor for depression : Theory and evidence. Psychological Review, 91(3), 347-374.

Peterson, C., Seligman, M.E. P. \& VAillant, G.E. (1988). Pessimistic explanatory style is a risk factor for physical illness : A thirty-five year longitudinal study. Journal of Personality and Social Psychology, 55, 23-27.

Peterson, C., Semmel, A., Von Baeyer, C., Abramson, L.Y., Metalsky, G.I. \& SELIGMAN, M.E. P. (1982). The Attributional Style Questionnaire. Cognitive Therapy and Research, 6(3), 287-300.

Peterson, C. \& SteEN, T.A. (2002). Optimistic Explanatory Style. In C.R. Snyder \& S.J. Lopez (Eds), Handbook of Positive Psychology (pp. 244-256). Oxford : University Press.

PETERSON, C. \& VAIDYA, R.S. (2001). Explanatory style, expectations, and depressive symptoms. Personality and Individual Differences, 31 (7), 1217-1223.

Peterson, C. \& Villanova, P. (1988). An expanded attributional style questionnaire. Journal of Abnormal Psychology, 97, 87-89.

PRAPAVESSIS, H. \& CARRON, A.V. (1988). Learned helplessness in sport. The Sport Psychologist, 2, 189-201. 
Proudfoot, J.G., CORR, P.J., GueSt, D.E. \& GrAy, J.A. (2001). The development and evaluation of a scale to measure occupational style in financial services sector. Personality and Individual Differences, 30, 259-270.

RADKe-YARROW, M., Belmont, B., NotTelman, E. \& BotTOMly, L. (1990). Young children's self-conceptions : Origins in the natural discourse of depressed and normal mothers and their children. In D. Cicchetti \& M. Beeghly (Eds.), The self in transition. Chicago : University of Chicago Press.

RASCLE, N. \& IRACHABAL, S. (2001). Médiateurs et modérateurs : implications théoriques et méthodologiques dans le domaine du stress et de la psychologie de la santé. Le Travail Humain, 64, 97-118.

REIVICH, K. (1995). The measurement of explanatory style. In G.M. Buchanan \& M.E. P. Seligman (Eds), Explanatory Style (pp. 21-47). Hillsdale, NJ : Lawrence Erlbaum Associates.

ReIVICH, K.J. \& SELIGMAN, M.E. P. (1991). The forced-choice Attributional Style Questionnaire. Unpublished data, University of Pennsylvania, Philadelphia.

RETTEW, D. \& REIVICH, K. (1995). Sports and Explanatory Style. In : G.M. Buchanan \& M.E. P. Seligman (Eds), Explanatory Style (pp. 173-185). Hillsdale, NJ : Lawrence Erlbaum Associates.

RIC, F. (1996). L'impuissance acquise (learned helplessness) chez l'être humain : une présentation théorique, L'Année psychologique, 4, PUF, 677-702.

RIC, F. (2001). Privation de contrôle et traitement de l'information: critique de l'approche de la motivation pour le contrôle et propositions alternatives. L'année Psychologique, 101, 349-369.

Roth, S. (1980). A revised model of learned helplessness in humans. Journal of Personality 48(1), 103-133.

SARRAZIN, P. \& GUILLET, E. (2001). « Mais pourquoi ne se réinscrivent-ils plus !» Variables et processus de l'abandon sportif. In F. CURY \& P. Sarrazin (Eds.), Théories de la motivation et pratiques sportives: état des recherches, pp. 223-254. Paris : PUF.

SCHULMAN, P. (1995). Explanatory style and achievement in school and work. In G.M. Buchanan \& M.E. P. Seligman (Eds), Explanatory style (pp. 159-171). Hillsdale, NJ : Erlbaum.

Schulman, P., KeITH, D. \& SEligman, M.E. P. (1991). Is optimism heritable? A study of twins. Behavior Research and Therapy, 31, 569-574.

Seligman, M.E. P. (1975). Helplessness : On depression, development and death. San Francisco : Freeman.

SELIGMAN, M.E. P. (1991). Learned optimism. New York : Knopf.

SELIGMAN, M.E. P. (1994). Apprendre l'Optimisme. Paris : InterEditions.

SELIGMAN, M.E. P. (2002). Authentic happiness : New positive psychology to realize your potential for lasting fulfilment. New York: Free Press.

Seligman, M.E. P., Abramson, L.Y., SEMmel, A. \& Von BAeYer, C. (1979). Depressive attributional style. Journal of Abnormal Psychology, 88, 242-247.

SELIGMAN, M.E. P. \& MAIER, S.F. (1967). Failure to escape traumatic shock. Journal of Experimental Psychology, 74, 1-9. 
Seligman, M.E. P., Nolen-Hoeksema, S., Thornton, K.M., Thornton, N. (1990). Explanatory style as a mechanism of disappointing athletic performance. Psychological Science, 1, 143-146.

SHAVER, K.G. (1975). An introduction to attribution processes. Cambridge, MA: Winthrop.

SILVER, R.L., WORTMAN, C.B. \& KLOS, D.S. (1982). Cognitions, affects, and behavior following uncontrollable outcomes: A response to current helplessness research. Journal of Personality, 50, 481-514.

Simmons, R.G., BLyTH, D.A., Van CleAVE, E.F. \& Bush, D.M. (1979). Entry into early adolescence : The impact of school structure, puberty, and early dating on selfesteem. American Sociological Review, 44, 948-967.

TAYLOR, S.E. \& BROWN, J.D. (1988). Illusion and well-being : A social psychological perspective on mental health. Psychological Bulletin, 103, 193-210.

VALLERAND, R.J. (1997). Toward a Hierarchical model of intrinsic and extrinsic motivation. In M.P. Zanna, Advances in Experimental Social Psychology (Vol. 29, pp. 271-360). New York: Academic Press.

VALlerand, R.J. \& GROUZET, F. (2001). Pour un modèle hiérarchique de la motivation intrinsèque et extrinsèque dans les pratiques sportives et l'activité physique. In F. Cury \& P. Sarrazin (Eds), Théories de la motivation et pratiques sportives : état des recherches (pp. 57-95). Paris : PUF.

WEINER, B. (1974). Achievement motivation and attribution theory. Morristown : General Learning Press.

WEINER, B. (1985). An attributional theory of achievement motivation and emotion. Psychological Review, 92, 548-573.

WORTMAN, C.B. \& BREHM, J.W. (1975). Response to uncontrollable outcomes : An integration of reactance theory and the learned helplessness model. In L. Berkowitz (Ed.), Advances in experimental social psychology (Vol. 8). New York: Academic Press.

ZIEGLER, D.J. \& HAWLEY, J.L. (2001). Relation of irrational thinking and the pessimistic explanatory style. Psychological Reports, 88, 483-488.

ZULlOW, H.M. (1995). Pessimistic rumination in American politics and society. In G.M. Buchanan \& M.E. P. Seligman (Eds.), Explanatory style (pp. 21-48). Hillsdale, NJ : Erlbaum. 